\title{
DESIGN OF A HIGH ALTITUDE LONG ENDURANCE FLYING-WING SOLAR-POWERED UNMANNED AIR VEHICLE
}

\author{
A. A. Alsahlani, L. J. Johnston, and P. A. Atcliffe \\ University of Salford \\ School of Computing, Science and Engineering \\ Salford, Greater Manchester M5 4WT, U.K.
}

\begin{abstract}
The low-Reynolds number environment of high-altitude flight places severe demands on the aerodynamic design and stability and control of a high altitude, long endurance (HALE) unmanned air vehicle (UAV). The aerodynamic efficiency of a flying-wing configuration makes it an attractive design option for such an application and is investigated in the present work. The proposed configuration has a high-aspect ratio, swept-wing planform, the wing sweep being necessary to provide an adequate moment arm for outboard longitudinal and lateral control surfaces. A design optimization framework is developed under a MATLAB environment, combining aerodynamic, structural, and stability analysis. Low-order analysis tools are employed to facilitate efficient computations, which is important when there are multiple optimization loops for the various engineering analyses. In particular, a vortex-lattice method is used to compute the wing planform aerodynamics, coupled to a twodimensional (2D) panel method to derive aerofoil sectional characteristics. Integral boundary-layer methods are coupled to the panel method in order to predict flow separation boundaries during the design iterations. A quasi-analytical method is adapted for application to flyingwing configurations to predict the wing weight and a linear finite-beam element approach is used for structural analysis of the wing-box. Stability is a particular concern in the low-density environment of high-altitude flight for flying-wing aircraft and so provision of adequate directional stability and control power forms part of the optimization process. At present, a modified Genetic Algorithm is used in all of the optimization loops. Each of the low-order engineering analysis tools is validated using higher-order methods to provide confidence in the use of these computationally-efficient tools in the present design-optimization framework. This paper includes the results of employing the present optimization tools in the design of a HALE, flying-wing UAV to indicate that this is a viable design configuration option.
\end{abstract}

(C) The authors, published by EDP Sciences. This is an Open Access article distributed under the terms of the Creative Commons Attribution License 4.0 (http://creativecommons.org/licenses/by/4.0/). 


\section{INTRODUCTION}

High-altitude aircraft flying in the stratosphere (around 20-30-kilometer altitude) can provide a useful platform for sensors to support a range of military and civilian surveillance tasks. These can include real-time monitoring of seismic risks or volcanic areas, early forest fire detection, border security surveillance, pipe-line and power-line surveys, telecommunication services, agriculture monitoring, etc. [1]. By flying at such high altitudes, the aircraft can see or cover large geographical areas at wide angles and, in addition, the altitude offers some protection in terms of possible interception by hostile vehicles. Although a number of research and operational high-altitude aircraft have been developed (including the Lockheed U-2, Helios, Zephyr, and Global Hawk) [2, 3], there still remain significant challenges in the design and operation of HALE aircraft, particularly, in respect to their payload capability.

To date, the majority of existing HA UAVs have been of conventional wing/ fuselage/tail/fin configuration. There is currently research being undertaken into the use of Blended-Wing Body configurations for UAV applications because of their perceived advantages in terms of aerodynamic and structural efficiency [4]. A true flying-wing is perhaps the most aerodynamically-efficient aircraft configuration but, to date, has not been investigated in any detail for possible application to HA UAVs. Such a configuration would require a moderate amount of wing sweep in order to generate stability in flight and to provide adequate control power for manoeuvring purposes. One objective of the present research work is to investigate the design and optimization of a swept, true flying-wing configuration for application to HA UAV operations. A swept, flying-wing configuration is to be studied for operation at these flight conditions, designed to meet a particular mission requirement. Specific topics to be considered will be development of a fast and accurate-as-possible multidisciplinary optimization tool able to design and optimize such a flying wing HALE UAV.

\section{METHODOLOGY}

The methodology which is adopted in order to complete the present project will involve the following steps.

1. Develop a multidisciplinary optimization tool which should have the following characteristics:

(a) computationally fast as possible with sufficient accuracy of solution;

(b) employ inviscid and viscous computational fluid dynamics (quasi-threedimensional CFD); 
(c) include the influence of structural elasticity and weight prediction; and (d) evaluate stability of the vehicle.

2. Evaluate the initial design according to a mathematical model for a solar powered aircraft to meet particular mission requirements which is 17kilometer altitude, 6-month endurance (March 1 to September 1) operating at 31-newton latitude (south of Iraq) with 50-kilogram payload for early forest fire detection purpose.

3. Apply to the flying-wing configuration for the specified mission profile and find an optimal design solution.

\section{AERODYNAMIC ANALYSIS}

Low-order analysis tools are employed to facilitate efficient computations, which is important when there are multiple optimization loops for the various engineering analyses. This module consists of two parts: a three-dimensional (3D) inviscid-flow model (Vortex Lattice Method, VLM) and a 2D viscid/inviscid flow solver. Additional supplementary tools are employed such as an aerofoil section geometry generator. The Tornado VLM is used to evaluate the lift force and induced drag for specific wing geometry. A 2D panel method, coupled with an integral boundary-layer method is used to evaluate $2 \mathrm{D}$ profile drag in a stripwise sense. Spanwise integration then leads to evaluation of the profile drag for the entire wing (Quasi-3D Aerodynamic Solver). The computational methods are developed and written under a MATLAB environment.

\subsection{Two-Dimensional Viscid/Inviscid Module}

In the present application of the incompressible flow around an aerofoil section, viscous effects are important only in the boundary-layer regions adjacent to the aerofoil surface. In this region, the governing Reynolds-averaged Navier-Stokes equations can be approximated by the so-called boundary-layer equations. An inviscid-flow model can be used outside this region [5]. A coupling is required between the inviscid-flow and boundary-layer regions. The boundary-layer calculations begin at the stagnation point at the aerofoil leading edge, with separate calculations for the upper and lower surface flows. The viscous-flow module consists of the following components:

I Laminar Flow Field: this field begins from the stagnation point near to the leading edge to the transition point from laminar to turbulent flow field. Thwaites' model is used to solve the momentum integral equation [5]. 
Additional investigation is added to predict the laminar separation which is considered as a transition point $[6,7]$.

II Transition Point: Michel's criterion is used to indicate the transition point from laminar to turbulent flow field [6]. The idea of this criterion is that the transition starts at specific local Reynolds number depending on the pressure gradient imposed on the boundary layer by the inviscid flow and the surface roughness [5].

III Turbulent Flow Field: after laminar separation or beyond the transition point, the boundary layer becomes turbulent. Head's method is used to predict the turbulent boundary layer development, using a number of semiempirical correlations of experimental data in addition to the momentum integral equation [6]. This model also checks whether separation is occurring; if not, the process will continue to the trailing edge.

The outputs from this calculation are momentum thickness, shape factor, skin friction coefficient, and displacement thickness for each panel. The tool calculates the profile drag (pressure and friction drag) by using the Squire-Young formula for the upper and lower surface (for more detail, see [5]).

\subsection{Quasi-Three-Dimensional Aerodynamic Solver}

In this method, the wing induced drag is evaluated using the Trefftz plane analysis (VLM), whereas the profile drag is evaluated using the strip theory [8]. The wing geometry is divided into several $2 \mathrm{D}$ spanwise wing sections, and by using the effective velocity and effective angle of attack, the aerodynamic forces on each segment will be evaluated. Strip Method procedure is detailed in [9]. The quasi-3D solver can be divided into three main steps as shown in Fig. 1.

Step One: Tornado VLM is used to evaluate the spanwise lift distribution and induced drag of the given wing geometry and flight state condition. The spanwise lift distribution can be interpolated for each certain strip, in addition to perform other calculations which are required to the next steps for each strip, such as strip planform area, local angle of attack, and chord length $c$.

Step Two: In this step, the effective angle of attack $\left(\alpha_{\text {eff }}\right)$ and profile drag $\left(C_{\text {deff }}\right)$, for a given local lift coefficient (from the first step) in each strip, will be calculated by using $2 \mathrm{D}$ aerodynamic characteristics of the wing section (aerofoil) by applying sweep theory. It means that the airspeed $V_{\infty}$ and aerofoil shape and other geometric parameters that are involved in the calculation should be based on the direction perpendicular to the sweep 


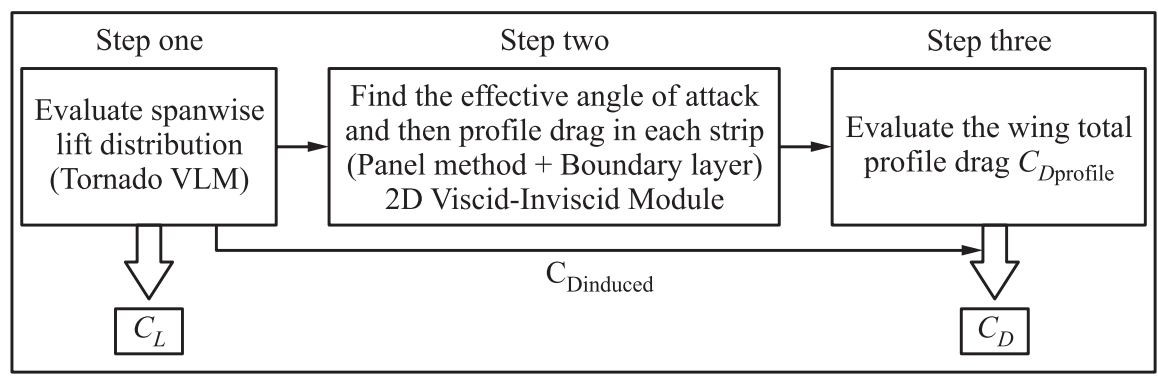

Figure 1 Quasi-3D solver procedure

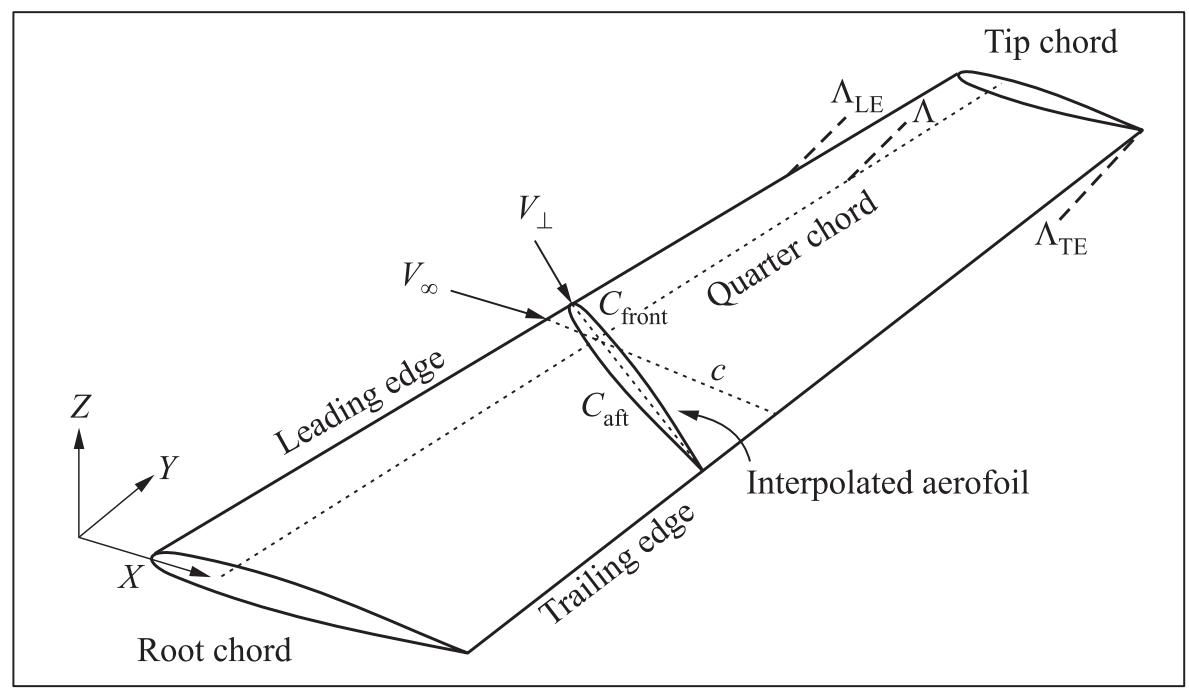

Figure 2 Aerofoil section perpendiculars to sweep line of a tapered wing $\left(C_{\text {front }}\right.$ $=\left(\sin \left(\pi / 2-\Lambda_{\mathrm{LE}}\right) / \sin \left(\pi / 2+\Lambda_{\mathrm{LE}}-\Lambda\right)\right) c / 4$ and $C_{\mathrm{aft}}=\left(\sin \left(\pi / 2-\Lambda_{\mathrm{TE}}\right) / \sin \left(\pi / 2+\Lambda_{\mathrm{TE}}\right.\right.$ $-\Lambda)) \cdot 3 c / 4)$

line as shown in Fig. 2. The sweep line can be the quarter chord sweep line while the flight speed is in subsonic range [10]. So, the perpendicular airspeed $V_{\perp}$ and perpendicular chord $c_{\perp}$ will be used instead of $V_{\infty}$ and $c$ as

$$
V_{\perp}=V_{\infty} \cos \Lambda ; \quad c_{\perp}=C_{\mathrm{aft}}+C_{\text {front }}
$$

where $C_{\text {aft }}$ and $C_{\text {front }}$ are shown in Fig. 2.

For the tapered swept wing case, the aerofoil which lies perpendicular to the sweep line, can be interpolated from its two neighboring aerofoils as shown in Fig. 2. 


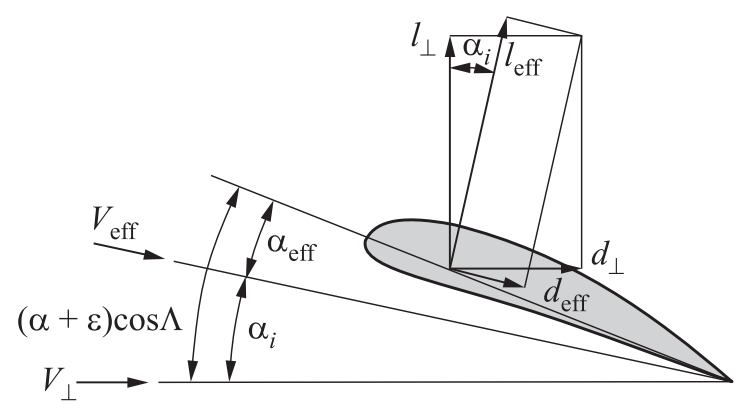

Figure 3 The angles and forces present in the quasi-3D method [9]

The corresponding lift coefficient $C_{l_{\perp}}$ is found using the sweep angle and the local lift coefficient $C_{l}$, which is determined from the lift distribution calculated by the VLM. So, it can be written as

$$
C_{l \perp}=C_{l} \sec ^{2} \Lambda
$$

Figure 3 shows the forces and angles at each wing section. The effective lift force can be given as

$$
l_{\mathrm{eff}}=\frac{l_{\perp}+d_{\mathrm{eff}} \sin \alpha_{i}}{\cos \alpha_{i}}
$$

or by using lift and drag coefficient instead of using the forces, this becomes:

$$
C_{l \mathrm{eff}}=\frac{C_{l \perp} \cos ^{2} \alpha_{i}+C_{d \mathrm{eff}} \sin \alpha_{i}}{\cos \alpha_{i}} .
$$

The effective Reynolds number can be evaluated by

$$
\operatorname{Re}_{\mathrm{eff}}=\operatorname{Re}_{\infty} \frac{V_{\mathrm{eff}} c_{\perp}}{V_{\infty} \bar{c}} .
$$

So, the steps which are followed in order to find the effective angle of attack and profile drag are shown in Fig. 4.

Step Three: The wing total profile drag coefficient $C_{D \text { prof }}$ is calculated in this step based on section profile strips $C_{D \text { prof }}$ which is calculated from the previous step as following:

$$
C_{D \text { prof }}=\frac{2}{s} \int_{0}^{b / 2} C_{d \text { prof }} c d y .
$$




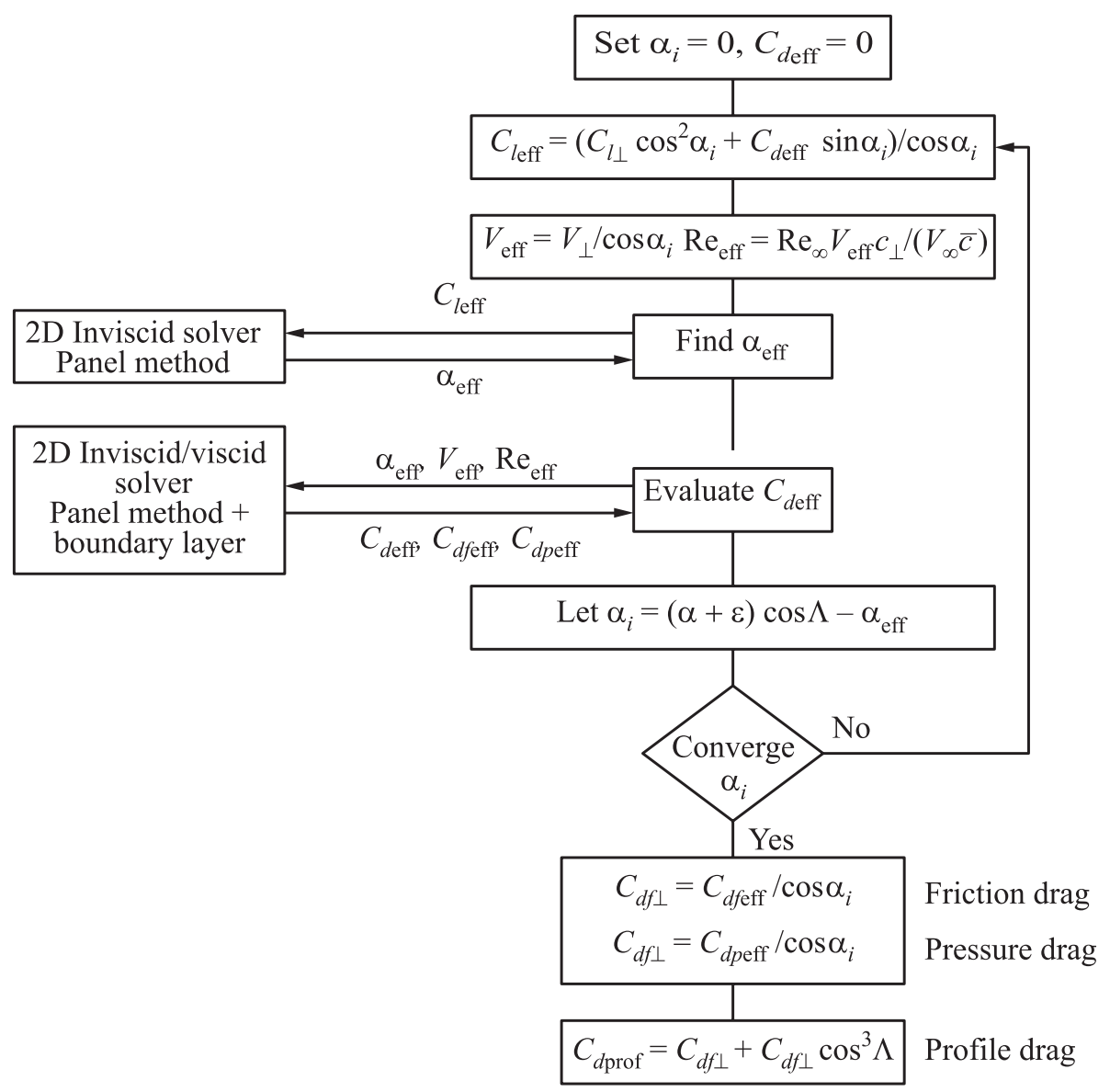

Figure 4 Steps of finding the effective angle of attack $\left(\alpha_{\text {eff }}\right)$ and profile drag

So, the total drag coefficient can be determined as a sum of wing profile drag coefficient plus the wing induced drag coefficient:

$$
C_{D}=C_{D \text { prof }}+C_{D i}
$$

This solver has been validated with two experimental cases operating at low Reynolds number which are shown in Fig. 5. The experimental data are from [11, 12]. The results show that this method can give a good approximation for the lift and drag coefficients in a short time (about $15 \mathrm{~s}$ in CORE i5 CPU $\mathrm{PC})$. 

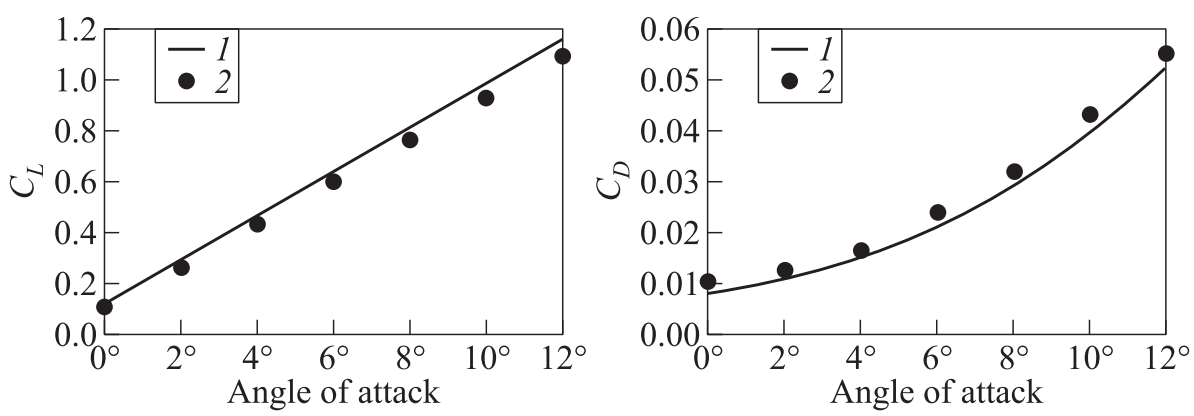

(a)
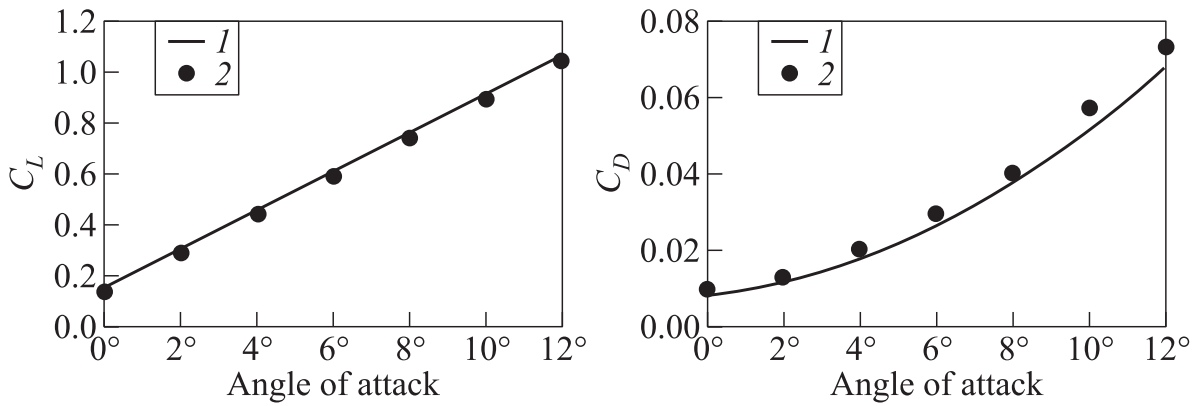

(b)

Figure 5 Validation of quasi-3D solver (1) with two experimental cases (2), $\mathrm{Re}=3.1 \cdot 10^{6}:(a)$ tapered wing validation (aspect ratio $=10$, taper ratio $=1 / 3$, and Dihedral $=0.687^{\circ}$ ); and $(b)$ swept tapered wing validation (aspect ratio $=6$, taper ratio $=1 / 2$, and Dihedral $\left.=0.974^{\circ}\right)$

\section{STRUCTURE ANALYSIS}

High altitude aircraft generally has an extreme span length, which suggests the aircraft is very flexible; so, it is very important to employ the elastic influence in the design tool. A MATLAB code has been written to size the wing box and to predict its weight by using a quasi-analytical method for a given aerodynamic load distribution (with other internal load such as weight and other inboard components: propulsion system, fuel cell, solar cell, avionic system, etc.) in addition to evaluating its deformation by using linear bending and torsion theory. The wing box is modeled by means of a $3 \mathrm{D}$ finite element beam (thin-walled beam) concentrated on the elastic axis of the wing such as shown in Fig. 6. This method is described in more detail in [11, 12]. The beam is discretized into elements, each element having two nodes. 


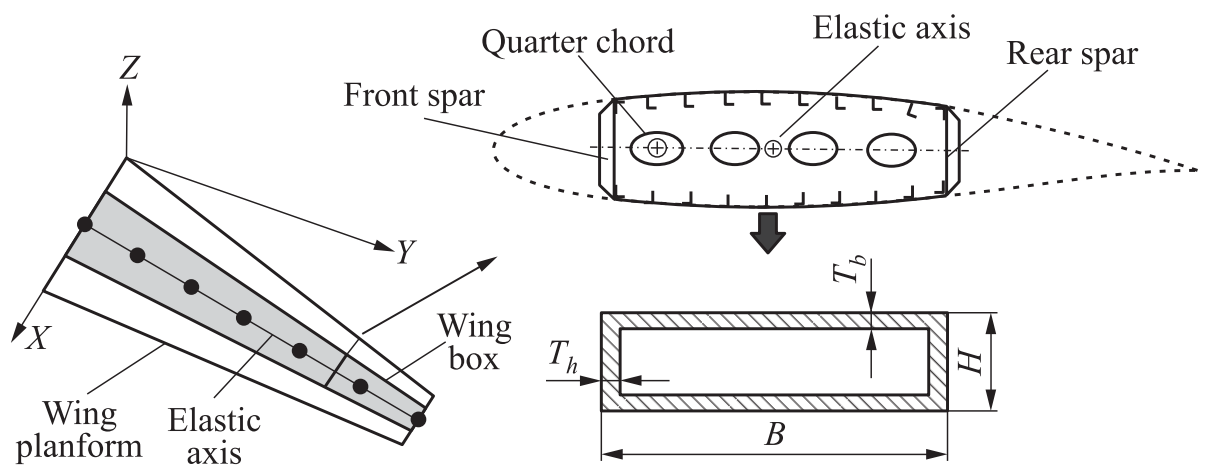

Figure 6 Sketch of the structural wing model and wing-box idealization

Each node has 6 degrees of freedom: three in translation and three in rotation. They are described by a local coordinate and then described by their rotation angles (sweep, dihedral, and twist) to transfer to the global coordinate system. Tornado Vortex Lattice is used to evaluate the lift forces in each element.

The tool starts with calculating the bending moment, shear force and torque in each element. Then, it finds the required thicknesses of the panel cross section ( $T_{b}$ and $T_{h}$, see Fig. 6), under a specific load factor assuming that the wing box resists all of the external force [11, 12]. The steps are shown in Fig. 7. Once the element cross section is sized, then the weight will be evaluated. In addition, the ribs' weight is evaluated from an empirical formula [12]. Element sizing includes spar web and equivalent skin representation, which is produced by summing the upper and lower skin panels, stringers, and the spar flange (see Fig. 6). The equivalent upper and lower wing panels resist bending and torsional loadings. The spar webs resist the vertical shear and torsional loads. The sizing process is iterated until the inertial relief effect is achieved.

The stiffness matrix $K$ for each element is then determined using the geometric and mechanical properties of the elements. The node displacement $U$ can be evaluated by the following relation [13]:

$$
F=K U
$$

where $F$ is the external load vectors; $K$ is the stiffness matrix; and $U$ is the displacement vector. After the sizing process, the deflection calculation will be performed which, in turn, requires the recalculation of the aerodynamic forces; so, the sizing is then reiterated until a quasi-static equilibrium between the structural and the aerodynamic forces is obtained. 


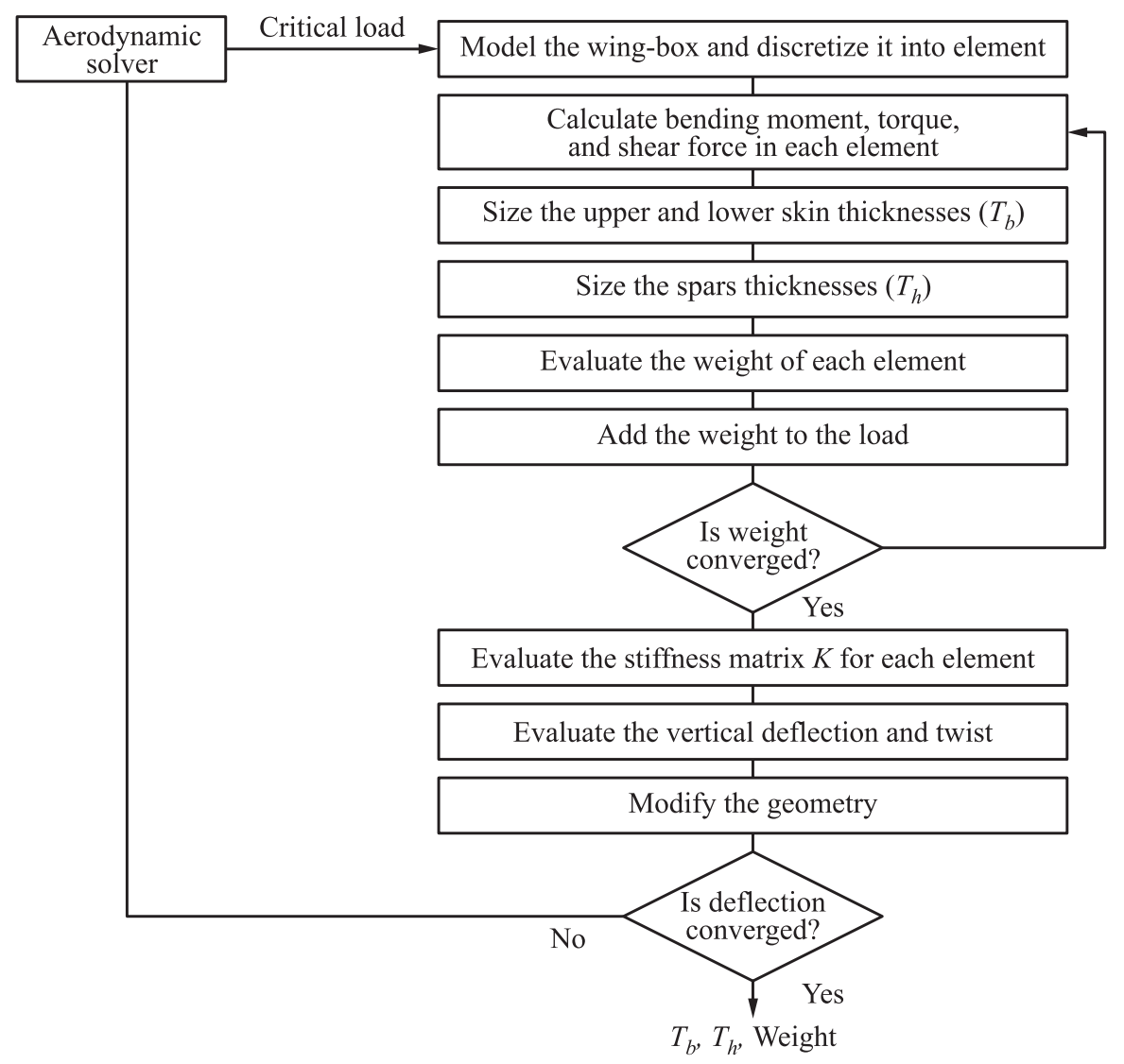

Figure 7 Wing-box sizing procedure

\section{STABILITY OF FLYING-WING AIRCRAFT}

Several design features specific to tailless flying-wing aircraft have been introduced, or suggested in the literature, to enhance vehicle control and stability. In terms of clean configuration geometry, these features are summarized as below and would need to be investigated for possible application to a HALE flying wing UAV:

- using reflexed aerofoil sections to achieve the pitching moment coefficient necessary to stabilize a tailless aircraft. However, this solution may reduce the lift at a certain angles of incidence and also reduce $C_{L \text { max }}$. The maximum camber location has a strong influence on the pitching moment and a slight effect on drag polar; so, it can be used to compensate for the lift 
reduction resulting from the use of a reflexed aerofoil section. The spanwise twist distribution does not give enough help in terms of stability with these types of aerofoil section $[14,15]$;

- selecting a suitable combination of sweep and twist distribution to generate zero pitching moment (with a slightly-reflexed aerofoil section) [16, 17];

- using a suitable dihedral angle (or dihedral distribution) could add some enhancement in the lateral direction [18]; and

- using winglets or C-wings to reduce induced drag and add further directional stability and control $[19,20]$.

Here, the HS 520 aerofoil section is selected for the whole wing sections because it has a very low pitching moment and can operate in the low Reynolds
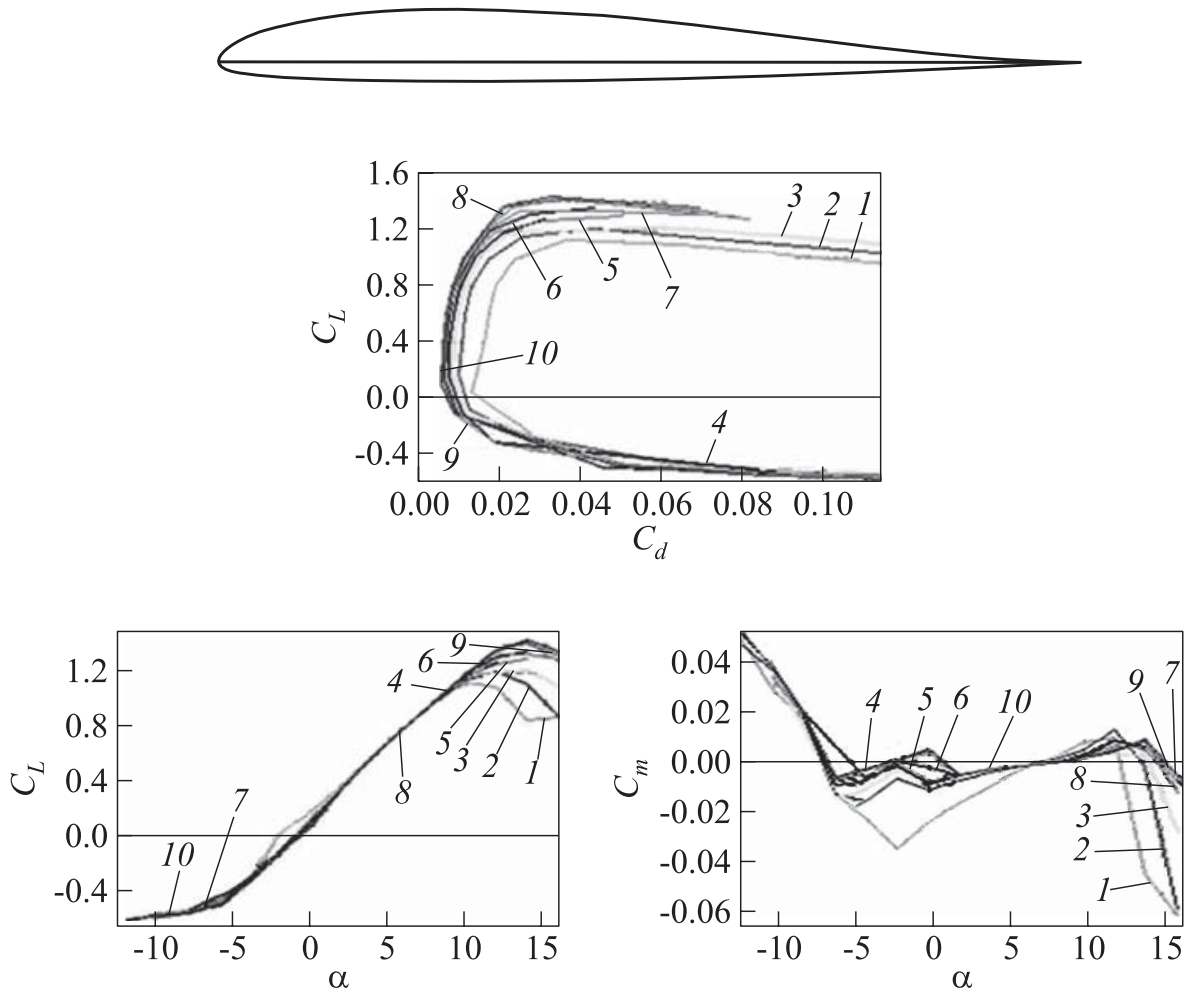

Figure 8 Aerofoil HS 520 at several Reynolds number values: $1-0.1 \cdot 10^{6} ; 2-$ $0.2 \cdot 10^{6} ; 3-0.3 \cdot 10^{6} ; 4-0.4 \cdot 10^{6} ; 5-0.5 \cdot 10^{6} ; 6-0.6 \cdot 10^{6} ; 7-0.7 \cdot 10^{6} ; 8-$ $0.8 \cdot 10^{6} ; 9-0.9 \cdot 10^{6} ;$ and $10-1.0 \cdot 10^{6}$ 
number environment with a good maximum lift coefficient as shown in Fig. 8, which is given by XFLR v6 (similar to Xfoil 2D CFD).

\section{PRECONCEPTUAL DESIGN}

A solar powered HALE UAV uses only the solar irradiance which is, in turn, dependent on the hour of the day, the day of the year, the latitude, and the position of the solar cell panels. For long endurance mission, the aircraft can fly continually if the energy collected during the daytime is enough to operate the aircraft during the whole day [21]. The energy and mass balance should be the starting point of the design. Motors, solar cell panels, fuel cells or batteries, and avionic system are dimensioned according to the required power but at the same time, they have weight which affects the gross weight that, in turn, affects the required power. There are two different approaches to achieve the conceptual design:

I The discrete and iterative approach: this is based on pure estimation for the first set of components (motors, solar cell panels, fuel cell or batteries, and avionic system) and from their weight, the total weight and power required can be estimated. This power is then compared with the previous selection and this process is performed iteratively until a converging solution is found. This approach requires more computational effort with good estimation models [22].

II An analytical and continuous approach: this consists of establishing all the relationships between all the components with analytical functions using their characteristics. This approach can provide directly a unique and optimal design but requires a robust mathematical model [22]. Noth et al. presented the methodology of designing a small solar powered aircraft, which was used to design the first prototype of Sky-Sailor [23]. In this paper, the second approach will be adopted with some modifications to the Noth methodology, particularly, in the structure mass and avionic mass and power modeling. Rizzo and Frediani proposed a structure mass estimation model which was obtained by data published for the NASA prototypes [24]. This function can be written in term of span length and aspect ratio to be implemented in the design tool:

$$
m_{\mathrm{af}}=1.548 b^{1.312} \mathrm{AR}^{-0.0046}[\mathrm{~kg}] \text {. }
$$

The mass and power of the system components can be estimated as a constant fraction of the structural mass, or the total mass, or of the power as shown in Fig. 9. Fuel cells with a lower mass to power density ratio are used instead of batteries $[1,24]$. 


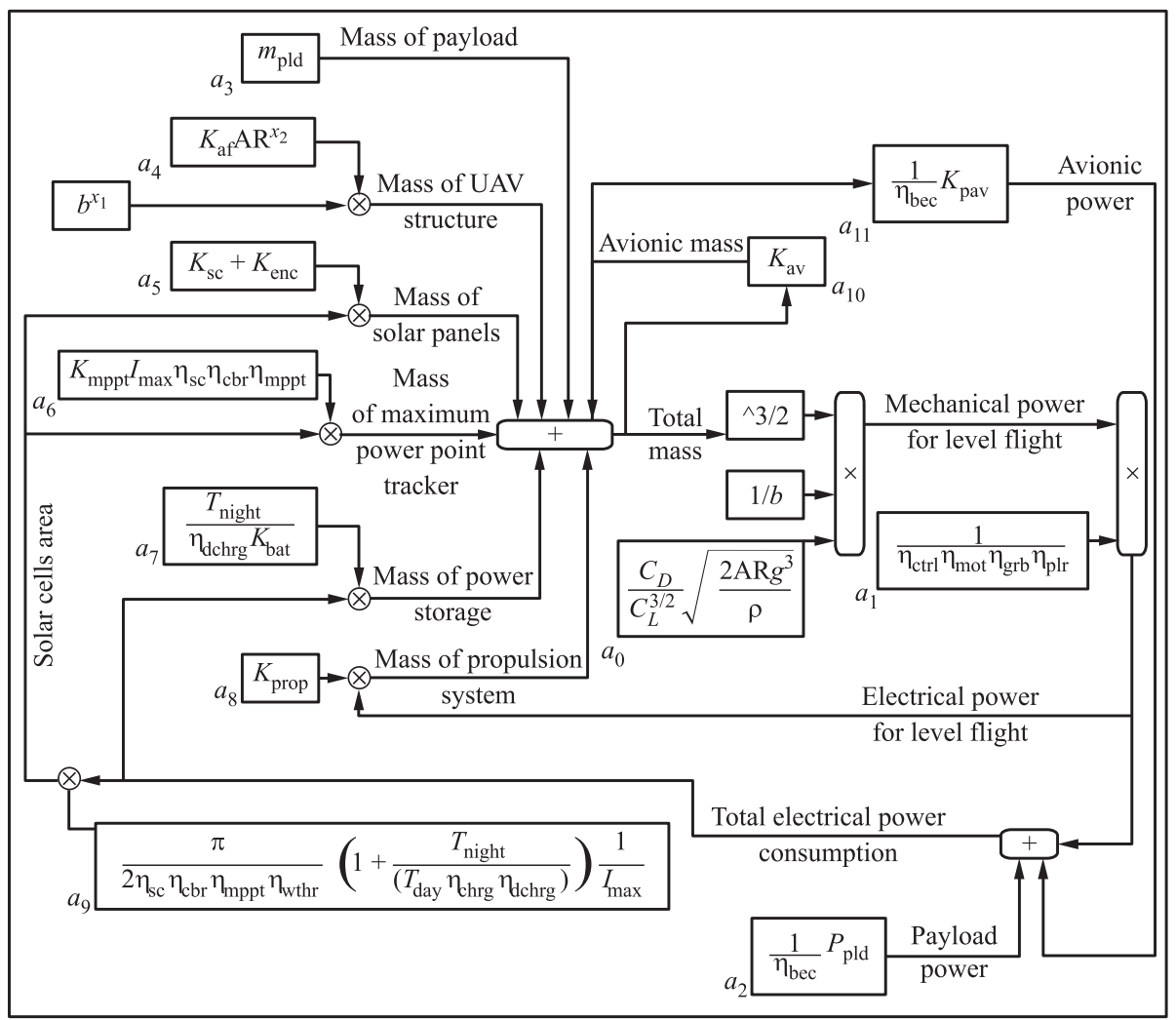

Figure 9 Schematic representation of the design methodology

The total mass $m$ of the aircraft is the sum of all the components such as:

$$
m=m_{\text {payload }}+m_{\mathrm{af}}+m_{\mathrm{sc}}+m_{\mathrm{mppt}}+m_{\text {avionic }}+m_{\mathrm{prop}}+m_{\mathrm{fc}} .
$$

For simplicity, variables $a_{i}$ can be used instead of a heavy equation as shown in Fig. 9 and after rearranging, the main equation becomes:

$$
\begin{gathered}
m=m \overbrace{\left[\left(a_{5}+a_{6}\right) a_{10} a_{11} a_{9}+a_{7} a_{10} a_{11}+a_{10}\right]}^{a_{12}} \\
+\frac{m^{3 / 2}}{b} \overbrace{\left[\left(a_{5}+a_{6}\right) a_{0} a_{1} a_{9}+a_{7} a_{0} a_{1}+a_{8} a_{0} a_{1}\right]}^{a_{13}} \\
+[b^{x_{1}} a_{4} \overbrace{\left.+a_{3}+\left(a_{5}+a_{6}\right) a_{2} a_{9}+a_{7} a_{2}\right]}^{a_{14}}
\end{gathered}
$$


where $a_{1}-a_{11}$ are defined in Fig. 9. This will lead to:

$$
m\left(1-a_{12}\right)-\frac{m^{3 / 2}}{b} a_{13}-\left(b^{x_{1}} a_{4}+a_{14}\right)=0 .
$$

The positive real root of the latter equation can be found for different span lengths $(b)$ and aspect ratios (AR), but the solution should be constrained so

Table 1 The constant parameters of the design

\begin{tabular}{|c|c|c|c|}
\hline Parameter & Value & Unit & Description \\
\hline$C_{L}$ & 0.9 & - & Aeroplane lift coefficient \\
\hline$C_{\mathrm{da}}$ & 0.01 & - & Aerofoil drag coefficient \\
\hline$e$ & 0.85 & - & Oswald's efficiency factor \\
\hline$I_{\max }$ & 974 & $\mathrm{~W} / \mathrm{m}^{2}$ & $\begin{array}{l}\text { Maximum irradiance (minimum (Imax) between } \\
\text { March } 1 \text { and October } 1 \text { ) at latitude } 31^{\circ} \text { south } \\
\text { of Iraq }[25]\end{array}$ \\
\hline$K_{\mathrm{fc}}$ & $550 \times 3600$ & $\mathrm{~J} / \mathrm{kg}$ & Energy density of fuel cell \\
\hline$K_{s c}$ & 0.25 & $\mathrm{~kg} / \mathrm{m}^{2}$ & Mass density of solar cells \\
\hline$K_{\text {enc }}$ & 0.01 & $\mathrm{~kg} / \mathrm{m}^{2}$ & Mass density of encapsulation \\
\hline$K_{\mathrm{mppt}}$ & 0.00047 & $\mathrm{~kg} / \mathrm{W}$ & Mass to power ratio of maximum power point tracker \\
\hline$K_{\text {prop }}$ & 0.0045 & $\mathrm{~kg} / \mathrm{W}$ & Mass to power ratio of propulsion unit \\
\hline$K_{\text {af }}$ & 1.548 & $\mathrm{~kg} / \mathrm{m}^{3}$ & Structural mass constant \\
\hline$\eta_{\text {cbr }}$ & $1^{*}$ & - & Efficiency of the curved solar panels \\
\hline$\eta_{\text {bec }}$ & $1^{*}$ & - & Efficiency of step-down converter \\
\hline$\eta_{\mathrm{sc}}$ & 0.31 & - & $\begin{array}{l}\text { Efficiency of solar cells (Triple-Junction } \\
\text { Epitaxial Liftoff Tabbed Solar Cell (MicroLink)) }\end{array}$ \\
\hline$\eta_{\text {chrg }}$ & $1^{*}$ & - & Efficiency of fuel cell charge \\
\hline$\eta_{\text {ctrl }}$ & 0.95 & - & Efficiency of motor controller \\
\hline$\eta_{\text {dchrg }}$ & 0.6 & - & Efficiency of fuel cell discharge \\
\hline$\eta_{\mathrm{grb}}$ & $1^{*}$ & - & Efficiency of gearbox \\
\hline$\eta_{\text {mot }}$ & 0.95 & - & Efficiency of motor \\
\hline$\eta_{\mathrm{mppt}}$ & $1^{*}$ & - & Efficiency of maximum power point tracker \\
\hline$\eta_{\mathrm{plr}}$ & 0.85 & - & Efficiency of propeller \\
\hline$x_{1}$ & 1.312 & - & Structural mass area exponent \\
\hline$x_{2}$ & -0.0046 & - & Structural mass aspect ratio exponent \\
\hline$K_{\text {av }}$ & 0.03 & - & Avionic weight fraction \\
\hline$K_{\text {pav }}$ & 6 & $\mathrm{~W} / \mathrm{kg}$ & Power-to-mass ratio of avionics \\
\hline$k_{\text {solmargin }}$ & $1^{*}$ & - & Irradiance margin factor \\
\hline$m_{\text {payload }}$ & 50 & $\mathrm{~kg}$ & Payload mass \\
\hline$P_{\text {payload }}$ & 850 & $\mathrm{~W}$ & Payload power consumption \\
\hline$\rho$ & 0.1382 & $\mathrm{~kg} / \mathrm{m}^{3}$ & Air density (at $17 \mathrm{~km})$ \\
\hline$T_{\text {day }}$ & $12 \times 3600$ & $\mathrm{~s}$ & Daytime duration \\
\hline
\end{tabular}



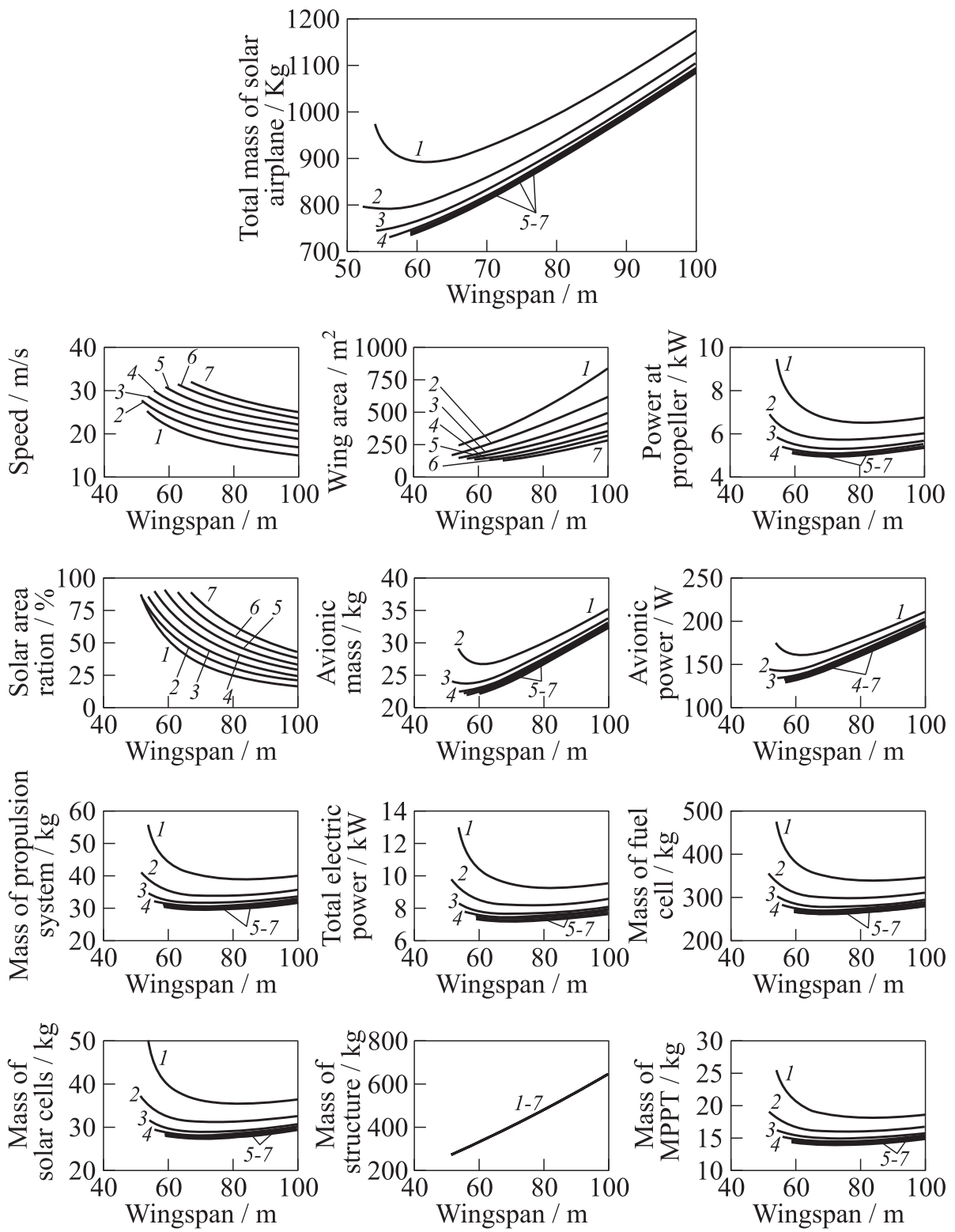

Figure 10 Possible design for the given mission requirements (MPPT - maximum power point tracker): $1-\mathrm{AR}=12 ; 2-16 ; 3-20 ; 4-24 ; 5-28 ; 6-32$; and $7-\mathrm{AR}=36$ 
Table 2 The main aeroplane characteristics

\begin{tabular}{lccl}
\hline Parameter & Value & Unit & \multicolumn{1}{c}{ Description } \\
\hline AR & 24 & - & Aspect ratio \\
$b$ & 56 & $\mathrm{~m}$ & Wingspan \\
$m$ & 731 & $\mathrm{~kg}$ & Total mass \\
$m_{\text {avionic }}$ & 22 & $\mathrm{~kg}$ & Avionic weight \\
$m_{\mathrm{fc}}$ & 277 & $\mathrm{~kg}$ & Fuel cell weight \\
$m_{\mathrm{prop}}$ & 31.5 & $\mathrm{~kg}$ & Propulsion system weight \\
$m_{\mathrm{af}}$ & 300 & $\mathrm{~kg}$ & Structure mass \\
$m_{\mathrm{sc}}$ & 29.3 & $\mathrm{~kg}$ & Mass of solar cells \\
$m_{\mathrm{mppt}}$ & 15 & $\mathrm{~kg}$ & Mass of maximum power point tracker \\
$S$ & 130.7 & $\mathrm{~m}$ & Platform area \\
$c_{\mathrm{av}}$ & 2.334 & $\mathrm{~m}$ & Mean chord \\
$V_{\infty}$ & 29.7 & $\mathrm{~m} / \mathrm{s}$ & Airspeed \\
\hline
\end{tabular}

that the value of the solar cell area is not more than the planform area. Table 1 shows the constant parameters of the components with the other mission requirements which are assumed still constant during the design process [23].

Applying this methodology to the design led to the possible shape of the aeroplane, for given mission requirements, as illustrated in Fig. 10.

It is clear that the span corresponding to the minimum aeroplane weight is about $56 \mathrm{~m}$ and aspect ratio 24 . The final choice guides the flight speed, structure weight, wing area, power and mass of fuel cells, avionic system, solar cells, avionic system, and propulsion system as shown in Table 2 . The initial geometry is now determined and need to be optimized to achieve the optimal flyable geometry.

\section{OPTIMIZATION}

A design optimization framework has been developed, under a MATLAB environment combining aerodynamic, structural, and stability analysis. A Canonical Genetic Algorithm has been used in the optimization process to vary the geometric variables until achieving the optimal design which is represented by the maximum or minimum fitness target. The optimizer code has been developed based on principle of genetic evolutionary processes as detailed in [26, 27]. Here, a single objective optimization process is used to find the minimum drag for a specific lift coefficient $\left(C_{L \text { reference }}=0.9\right)$ and static margin (not less than 0.05$)$. The final configuration should be stable statically and trimmed at the reference lift coefficient and at the mission altitude. Six electric motors are used and 


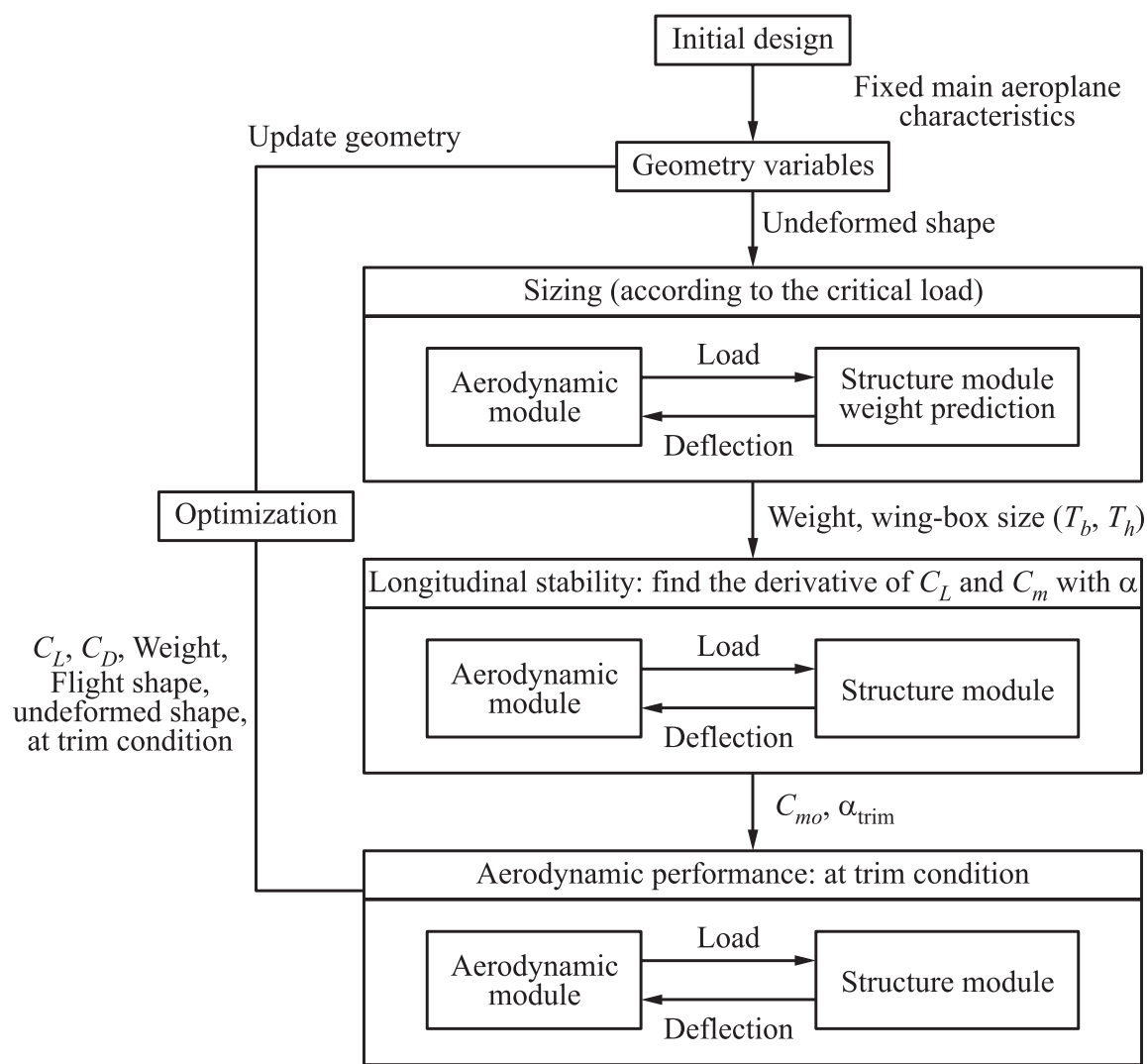

Figure 11 Optimization architecture

distributed along the span in addition to storage tanks of the fuel cells. The other fuel cell components and payload are assumed to be located at root chord and can be placed to adjust the center of gravity to achieve a specific lift coefficient. The communication shape among the disciplinary tools is presented in Fig. 11. A suitable mesh density is used to achieve a good sensitivity with varying the geometric variables for this configuration (5 chordwise $\times 39$ semispanwise panels are used for each half span or 390 panels for entire wing). Increasing the number of panels can lead to more accuracy, but at more computational cost.

The problem here is formulated for a constant area and wing span to vary the root chord, the sweep of each part of the wing $\left(\mathrm{Sw}_{1}\right.$ and $\left.\mathrm{Sw}_{2}\right)$, their taper ratio $\left(\mathrm{TR}_{1}\right.$ and $\left.\mathrm{TR}_{2}\right)$, length of the first partition $\left(b_{1}\right)$, and the twist distribution of the wing (Fig. 12). A static longitudinal stability only is considered now. 


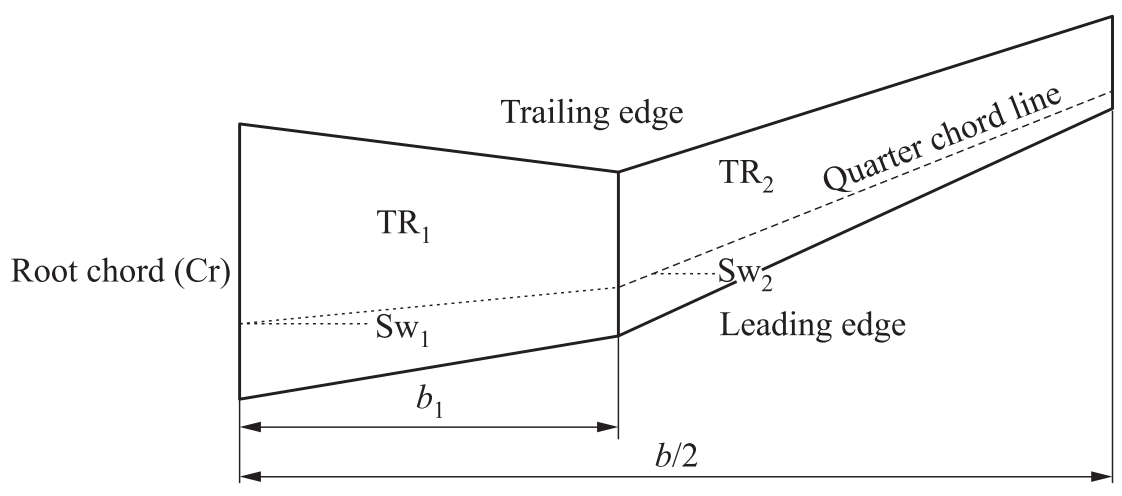

Figure 12 Design variable of the half wing

Further implementation such as lateral and directional static and dynamic stability will be implemented in the future work. This problem is formulated as follows:

Minimize: $C_{D}$

Variables: $\mathrm{TR}_{1}, \mathrm{TR}_{2}, \mathrm{Sw}_{1}, \mathrm{Sw}_{2}, \mathrm{Cr}, b_{1}$, twist distribution

Subject to $C_{L} \geq C_{L \text { reference, }} C_{m}=0$, no separation is allowed, $S=130.7 \mathrm{~m}^{2}$, $\operatorname{span}=56 \mathrm{~m}$

\section{OPTIMIZATION RESULTS}

The results, which are obtained from the optimization, show that it can achieve a flyable flying wing for the given mission and flight state. The aeroplane is trimmed at 11.7 degree angle of attack, 0.905 lift coefficient, and 0.0193 total drag coefficient. The gradient of the pitching moment coefficient is -0.053 and the zero lift pitching moment coefficient is approximately 0.0536, indicating a statically stable aeroplane in the longitudinal mode. The final configuration is shown in Fig. 13.

The sweep required to trim this aeroplane is about $8^{\circ}$ with the washout twist distribution shown in Fig. 14. Only the root section seems close to stall angle at cruise condition (see Fig. 8) while the washout twist distribution (see Fig. 14) can prevent stalling for the rest of the wing. Even if the tool does not predict any separation at any section at cruise condition, this would be investigated by a high-order CFD or by an experiment for further reliability in the future work. It is known that the structure deflection has an effect on the aeroplane geometry 


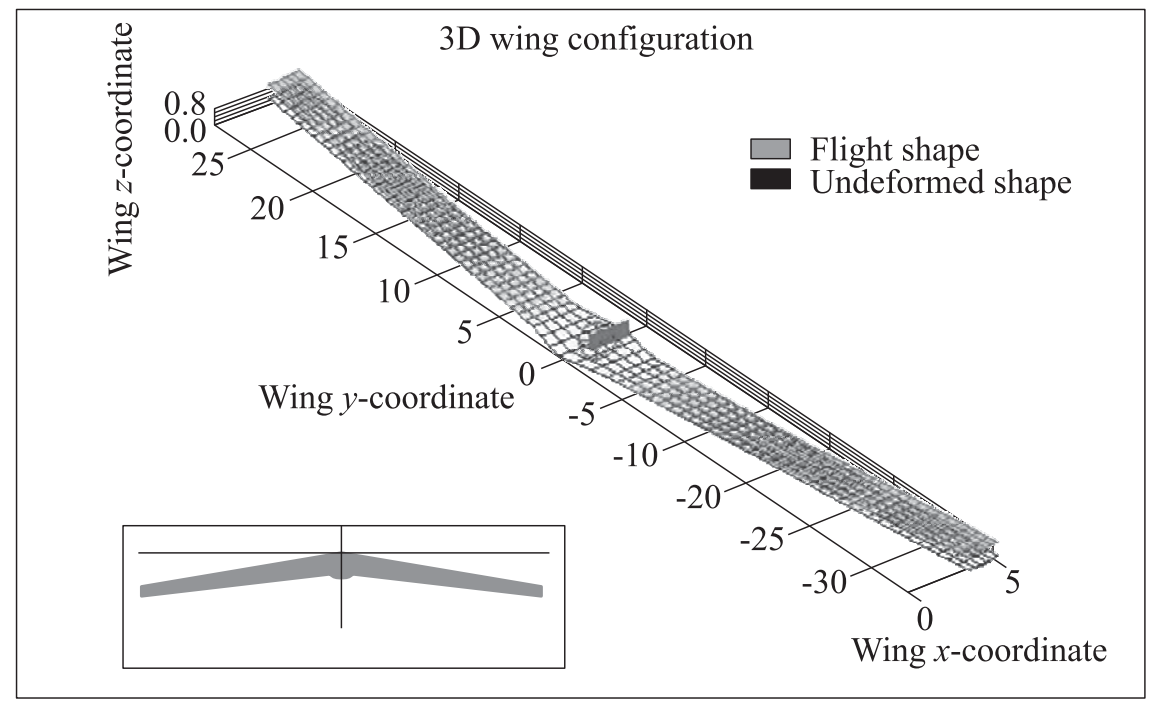

Figure 13 The final design at Cruise and Cruise off conditions: 1 - flight shape; and 2 - undeformed shape

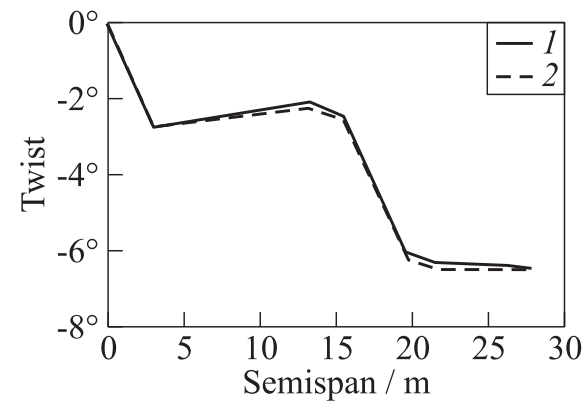

Figure 14 Twist distribution for flight (1) and undeformed shapes (2)

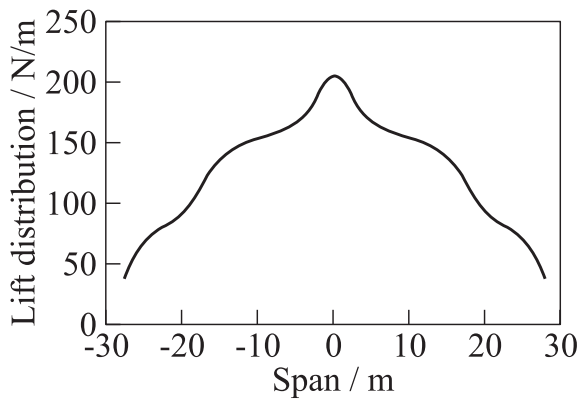

Figure 15 Lift distribution at trim condition

such as twisting in addition to increasing dihedral of the wing. The optimal undeformed wing shape is selected according to its flight shape performance while the optimization variables are for the undeformed shape. The structure behavior is depicted in Figs. 13 and 14. The lift force distributions are shown in Fig. 15 . 


\section{CONCLUDING REMARKS}

This paper presents a design and optimization framework employing aerodynamics and structure influence for a HALE, solar-powered flying wing UAV. By using modified low-order analysis tools that are employed to facilitate efficient computations for the various engineering analyses, a good approximation is achieved compared to the experimental data. Applying the flying-wing configuration in this tool leads to an aeroplane that can be trimmed to carry the designed payload for long endurance (6 months) at latitude $31 \mathrm{~N}$ and altitude $17 \mathrm{~km}$. As expected, the static longitudinal stability requires the design to be swept and twisted to give a moment arm behind the neutral axis to trim the aeroplane at the reference lift coefficient.

Future work to be undertaken includes analyzing the dynamic stability and control, using nonlinear structure behavior, using composite materials instead of a metallic wing structure, comparison between different configurations, and using multiobjective optimization to cover several objective targets.

\section{ACKNOWLEDGMENTS}

The authors would like to thank and dedicate this to Dr. L. J. Johnston who passed away during work on the present paper.

\section{REFERENCES}

1. Cestino, E., G. Frulla, and G. Romeo. 2007. Design of a high-altitude longendurance solar-powered unmanned air vehicle for multi-payload and operations. P. I. Mech. Eng. G J. Aer. Eng. 221(2):199-216.

2. Zdobysław, G., F. Andrzej, and W. Jacek. 1999. Design concept of a high-altitude long-endurance unmanned aerial vehicle. Aircraft Design 2(1):19-44.

3. Rapinett, A. 2009. Zephyr a high altitude long endurance unmanned air vehicle. Department of Physics, University of Surrey. MSc Thesis.

4. Cestino, E. 2006. Design of solar high altitude long endurance aircraft for multi payload \& operations. Aerosp. Sci. Technol. 10(6):541-550.

5. Wauquiez, C. 2009. Shape optimization of low speed airfoils using MATLAB and automatic differentiation. VDM Verlag. 68 p.

6. Moran, J. 1984. An introduction to theoretical and computational aerodynamics. Mineola, NY: Dover Publications, Inc. 466 p.

7. Katz, J., and A. Plotkin. 2001. Low-speed aerodynamics. Cambridge aerospace ser. Cambridge University Press. Vol. 13. 613 p. 
8. Mariens, J. 2012. Wing Shape multidisciplinary design optimization. Aerospace Engineering, Delft University of Technology. MSc Thesis.

9. Mariens, J., A. Elham, and M. van Tooren. 2014. Quasi-three-dimensional aerodynamic solver for multidisciplinary design optimization of lifting surfaces. J. Aircraft $51(2): 547-558$.

10. Obert, E. 2009. Aerodynamic design of transport aircraft. IOS PRESS. 656 p.

11. Ajaj, R. M., D. Smith, and A. T. Isikveren. 2013. A conceptual wing-box weight estimation model for transport aircraft. Aeronaut. J. 177(1191):533-551.

12. Torenbeek, E. 2013. Advanced aircraft design: Conceptual design, technology and optimization of subsonic civil airplanes. John Wiley \& Sons. 436 p.

13. Seywald, K. 2011. Wingbox mass prediction considering quasi-static nonlinear aeroelasticity. Technische Universität and Kungliga Tekniska högskolan. Bauhaus Luftfahrt. Diploma Thesis.

14. Buckstrom, A. 1979. The elements of tailless airplane design. Sport Aviation 3944.

15. Qin, N., A. Vavalle, A. Le Moigne, et al. 2004. Aerodynamic considerations of blended wing body aircraft. Prog. Aerosp. Sci. 40(6):321-343.

16. Li, P., B. Zhang, Y. Chen, et al. 2012. Aerodynamic design methodology for blended wing body transport. Chinese J. Aeronaut. 25(4):508-516.

17. Mader, C. A., and J.R. Martins. 2013. Stability-constrained aerodynamic shape optimization of flying wings. J. Aircraft 50(5):1431-1449.

18. Song, L., H. Jang, Y. Zhang, et al. 2014. Dihedral influence on lateral-directional dynamic stability on large aspect ratio tailless flying wing aircraft. Chinese J. Aeronaut. 27(5):1149-1155.

19. Bolsunovsky, A., N. Buzoverya, B. Gurevich, et al. 2001. Flying wing - problems and decisions. Aircraft Design 4(4):193-219.

20. Martinez-Val, R., E. Perez, P. Alfaro, and J. Perez. 2007. Conceptual design of a medium size flying wing. P. I. Mech. Eng. G J. Aer. Eng. 221(1):57-66.

21. Gao, X.-Z., Z. Hou, Z. Guo, J Liu, and X.-Q. Chen. 2013. Energy management strategy for solar-powered high-altitude long-endurance aircraft. Energ. Convers. Manage. 70:20-30.

22. Noth, A. 2008. Design of solar powered airplanes for continuous flight. ETH Zürich. $\mathrm{PhD}$ Thesis.

23. Noth, A., R. Siegwart, and W. Engel. 2008. Design of solar powered airplanes for continuous flight. 19 p.

24. Rizzo, E., and A. Frediani. 2008. A model for solar powered aircraft preliminary design. Aeronaut. J. 112(1128):57-78.

25. Tadros, M. T. Y., M. A. M. Mustafa, and M. Abdel-Wahab. 2014. Estimation of the global horizontal solar radiation in Iraq. Int. J. Emerging Technol. Adv. Eng. $4(8)$. 
26. Khan, F. A., P. Krammer, and D. Scholz. 2010. Preliminary aerodynamic investigation of box-wing configurations using low fidelity codes. DGLR Dtsch. Luft-und. Document ID: 161308 .

27. Khan, F. A. 2010. Prelimnary aerodynamic investigation of box-wing configrations using low fidelity code. Department of Space Science, Luleạ University of Technology. MSc Thesis. 